\title{
Projection of Constraint Based on Clustering
}

\author{
Jai Sharma. K, Chandra Mouliswara Reddy. P
}

\begin{abstract}
In this current framework, generally in on-line web site, purchasers can accounts in looking web site. They consumer login their record and examine the things. The, Recent summary report says that nice the good the nicer a part of business consumer area unit giving their phony survey among the great things. This might prompted negative shade among the item and it's been huge draw back among the administration aspect moreover, contributive tremendous total and that they try substantial misfortune in market. To defeat this issue, in projected framework, the consumer can provide the audit while not getting the things. It's primarily to dodge the phony purchasers among the things. The consumer survey framework needs to be overseen by the superior crew. This method wills progressively viable whereas contrasted with the past existing procedure.
\end{abstract}

Keywords: Clustering, Constraints, Data Processing, Framework

\section{INTRODUCTION}

Gathering study [1]-[5] sets up a basic branch of outfit learning. Precisely once differentiated and normal single agglomeration approaches, they'll facilitate totally different packing results into a sure along result that is increasingly precise moreover, stable. The gathering approach are often parceled into 2 stages: organization age and understanding work. the target of the organization age prepare is to induce contrastive social gathering individuals, whereas the target of the agreement work is to deliver a united batching game set up as properly as may well be allowable. beginning late, bunch outfits have grabbed a lot of thought, in light-weight of their confirming applications in several investigate domains [6]-[15], for example, data processing [6]-[8], image designing [9-14], then forth. For instance, Yu et al. [6] [7] planned Associate in Nursing filmable pack organization method for uproarious information processing. The proposed method [8] organized the weighted bundling gathering framework for common data processing. To determine.[9] associated the frightful grouping social function to manage high spacial objectives remote characteristic footage. Tsai et al. [10] planned a selfmasterminding map-based cluster gathering framework for multi-measured image bundling. Yu et al. [11] [12] thought of the way to apply mixture outfit approaches to manage handle biomolecular information. System discussed [13] explored the way to utilize gathering approach for microarray quality gathering recognizing proof. Zhuang et al. [14] organized the principled pack organization structure for changed malware and phishing web site distinctive proof.

Revised Manuscript Received on July 13, 2019.

Jai Sharma. K, Assistant Professor, Saveetha School of Engineering, SIMATS, Chennai

Chandra Mouliswara Reddy. P, UG scholar, Saveetha School of Engineering, SIMATS, Chennai.
Framework proposed [15] found way to pertain pack organization approach including perpetrator on-screen character space in internet primarily based life frameworks. With everything taken into consideration, amass gathering approaches are with success associated to varied zones, and have created extraordinary ends up in differing types of datasets.

Nonetheless, a few of limitations exist for traditional gathering amass approaches. (1) Few think about learning gave by specialists in unequivocal zones. (2) Few deem well ordered tips to manage high-dimensional information. (3) Most treat every organization half correspondingly nevertheless the manner that exact get-together individuals create clear duties to last output. (4) Whereas pairwise wants raise last result, few could unfavourably impact the result To handle these restrictions, we have a tendency to initially propose the semi-directed grouping equip dependent on picked necessity projection that area unit previous information given by specialists. Completely different weight regards area unit parcelled out to specific social function individuals as shown by different subsets of pairwise restrictions that augment the variable assortment of the gathering. (2) CESCP not merely gets the spasmodic mathematical space system to manage high-dimensional information, however furthermore occupations the restriction projection method to stipulate dimensional information into less-dimensional house. In sight of CESCP, we have a tendency to more propose the 2 fold weight semicoordinated furnish packing approach (DCECP) that joins two weight method: the essential weight process and also the filmable collecting half weight system. DCECP has the subsequent characteristics.

\section{LITERATURE SURVEY}

Mean shift agglomeration is probably a strong records point technique does not that does not want previous facts of clusters s well as would not constrain shape of clusters. But, being absolutely un attended, its performance suffers once the primary distance metric fails to capture the underlying cluster shape.

Cluster goal is to shape ensemble into searching for out unified cluster shape since more than one cluster systems acquired starting in reality specific datasets. Every cluster systems supply to unified cluster shape. The system finds the way of choosing correct cluster systems inside ensemble that is represented as cluster shape. In particular, shape of cluster takes preliminary depicted with the help of mixture of mathematician sharing, parameters of that calculable exploitation the rule named as expectation-maximization. Supported similarity contrast outcomes, the system has a propensity to endorse a contemporary approach, this is noted because of the fact the cluster based on distribution cluster 
form ensemble framework, to are searching for out the foremost representative unified cluster shape. We generally tend to then style a modern approach, the distributionprimarily based cluster form choice approach (DCSSS), to pick out a hard and speedy of cluster systems. In the long run, we usually tend to suggest the use of a distributionprimarily based normalized hypergraph lessen rule to get the last end result.

Cluster ensemble helps in gaining knowledge of domain that may be a crucial analysis consciousness. Goal of this is mixing a couple of clump answers in a completely suitable thanks to enhance the same old of the clump end result. In the course of this paper, we will be inclined to fashion a ultra-modern cluster ensemble system called AP2CE to handle demanding situations via datasets that are clangorous. AP2CE gets benefit of affinity propagation algorithmic application as well as additionally cut that is normalized for algorithmic program $\mathrm{N}$ reduce but conjointly contains features of such systems. Compared with historic cluster ensemble procedures, AP2CE is characterized by way of many residences.

Tumor bunching from bio-atomic information gives another approach to perform disease class revelation. In this paper, we propose a half and half fluffy group gathering system (HFCEF) for tumor bunching from malignant growth quality articulation information. Contrasted and customary group outfit system, HFCEF coordinates both the hard bunching and the delicate grouping into the group gathering structure. In particular, HFCEF first applies the fondness spread calculation (AP) to perform bunching on the quality measurement, and produces a lot of subspaces which are utilized to make a lot of new datasets. At that point, the fluffy enrollment work and the partiality proliferation calculation are embraced to create a lot of fluffy networks in the gathering. At long last, the standardized cut calculation is filled in as the agreement capacity to abridge the arrangement of fluffy grids and acquire the last outcome. The tests on disease quality articulation profiles demonstrates that the proposed structure functions admirably on bio-sub-atomic information, and gives progressively vigorous, steady and exact outcomes.

As a promising route for heterogeneous information investigation, agreement bunching has pulled in expanding consideration in ongoing decades. Among different incredible arrangements, the co-affiliation grid based strategies structure a milestone, which rethinks agreement grouping as a chart parcel issue. By the by, the moderately high existence complexities block it from wide genuine applications. We at long last stretch out SEC to address the difficulty emerging from deficient essential parcels, in view of which a column division plot for huge information bunching is proposed. Examinations on different genuine informational indexes in both outfit and multi-see bunching situations exhibit the prevalence of SEC over some cutting edge strategies. Specifically, SEC is by all accounts a promising possibility for enormous information grouping.

\section{SYSTEM ARCHITECTURE}

\subsection{Existing System}

In this present structure, generally in online website, customers will accounts in shopping page. They customer login their record and view the things. The, Recent graph report says that an enormous part of business customer are giving their fake examination among the remarkable things. This may instigated negative shade among the thing and it has been epic hindrance among the affiliation side Furthermore, contributing colossal aggregate and they confronting noteworthy disaster in market.

\subsection{Proposed System}

To squash this issue, in proposed framework, the client will give the survey without acquiring the things. It is on a very basic level to maintain a strategic distance from the phony clients among the things. The client outline framework ought to be controlled by the supervisory gathering. This structure wills dynamically down to earth compared to existing system.

\subsection{Modules}
$>$ Graphical User Interface
$>$ Admin Maintain the Products.
$>$ User Transaction.
$>$ Key generate \& Review sharing.
$>$ Dual sentiment analysis.
$>$ Performance Evaluation.

\subsubsection{Graphical User Interface}

First module of our proposed system is GUI. Essential process of consumer is transporting login to another window named as patron. It is for safety motive. Here first user enters login purchaser identity as well as mystery key. Username is checked also secret phrase is coordinate or not giant customer identification as well as valid secret key. Within the event that we input any invalid username or mystery key we cannot cross in login page to consumer page it's going to demonstrates mistake note. From unauthenticated consumer going into the login page to purchaser page. The system provides respectable safety to venture. Server includes client id as well as secret. Word server verifies validation of purchaser. System properly develops security $\mathrm{s}$ well as maintaining those unapproved patron goes in gadget. The system utilize JSP in order to make shape. The system approves login patron as well as server verification.

\subsubsection{Admin Maintain the Products}

Primary module of system is maintaining products. Essential position in case of Product owners mainly lies in transporting login page to Product proprietor page. On login page, user enters consumer identification as well as password. System checks for matching username as well as password. In case of invalid username or password errors message is popped. So we're stopping from unauthorized product owner getting in login page to product owner page. 
It offers a great protection in case of login. Server has user details as well as password server take a look at person authentication. Product owner updated merchandise. System improve security also prevents unauthorized people entering community. System uses JSP for growing design. Login user and server authentication is validated.

\subsubsection{User Transaction}

Fourth module in system is part of labor done inside database the board framework or identical framework in opposition to a database, and dealt with in a rational and reliable manner unfastened of different exchanges. An exchange for the maximum component speaks to any adjustment in database. Consumer will circulate the sum to provider.

\subsubsection{Key Generate \& Review Sharing}

System help customer to impart Insights as well as check substance is secure providing coverage. An abnormal range generator is bodily gadget intended for producing photographs which is sensibly predicted advanced by arbitrary danger. Keys are created to statistics. Key is special for each component at every stage.

\subsubsection{Dual Sentiment Analysis}

Here majority of first preparing tests can be turned around to alternate extremes. We allude to them as "unique preparing set" and "turned around preparing set" individually. In our information development strategy, there is a balanced correspondence between the first and switched surveys. The classifier is prepared by boosting a blend of the probabilities of the first and turned around preparing tests. This procedure is called double preparing (DT).

\section{SYSTEM ARCHITECTURE}

The frameworks engineer builds up the essential structure of the framework the system proposed consists of SemiSupervised Clustering Algorithm as well as Clustering Sentiment calculation (CSA) is a double expectation (DP) separately, to utilize the first and switched tests two by two for preparing a factual classifier and make forecasts. To utilize the audits tests two by two for preparing a measurable classifier and make expectations. Forecasts are made by thinking about different sides of one survey.

\section{Points of interest:}

- Avoiding phony Reviews.

- Maintain with Security for Each audit.

- Showing Survey through Graph of Particular Product.

- Sentiment on elements (Reviews, Rating... and so forth.)

- These surveys are developing deals.

- Improve The Sales.

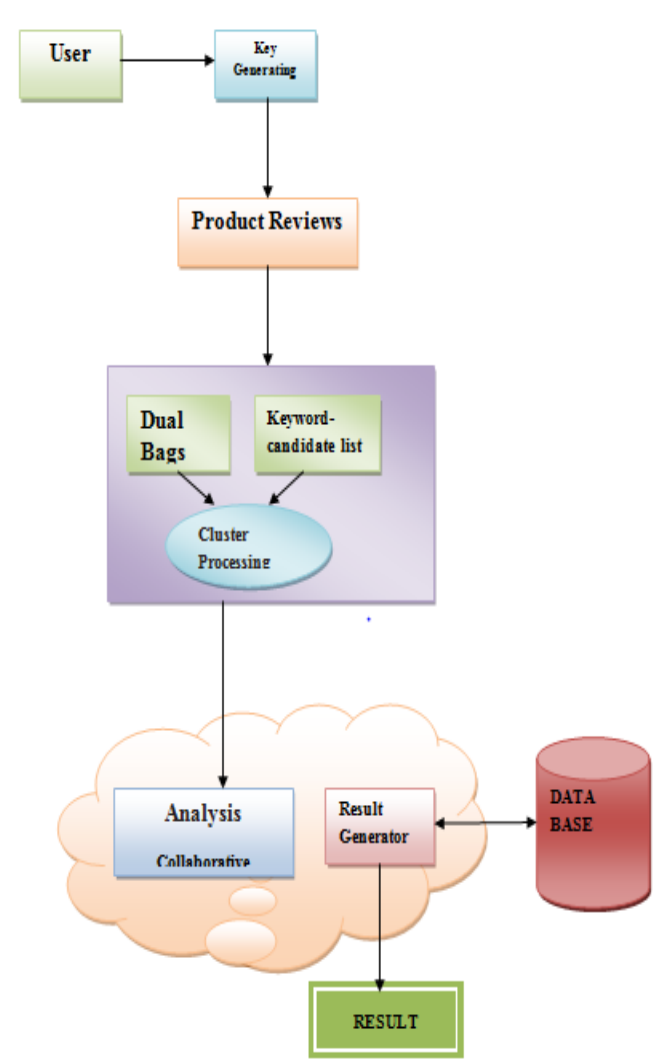

Fig. 1 System Architecture

\section{PERFORMANCE EVALUATION}

On this assignment what we are able to carry out implies, assessment of whole datasets, catchphrases, also tables, demands, requestors as well as quantity the system is able to perform productively without making delay to cease client.

On this module is applied to assist to the client to impart their Insights and take a look at their substance is in safe moreover giving insurance. An irregular wide variety generator that is used here stands as physical system that supply succession of pictures that is not sensibly known advanced with the help of arbitrary hazard. Generating keys is procedure for key creation. Key is unique for each component whilst on season among gets. Trials are developed dependent mistreatment fifteen real datasets yet because the system win related to ends. Acceptable parameter esteems can improve the presentation of the projected methodology. The versatile organization half weight procedure assumes a major job in DCECP. 


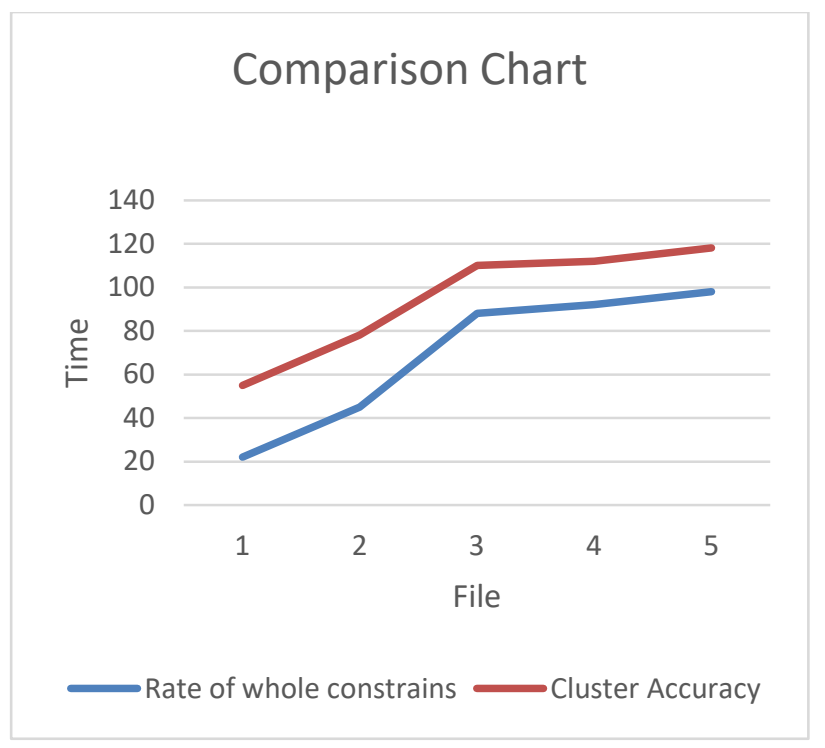

Fig. 2 Comparison Chart

\section{CONCLUSION AND FUTURE WORK}

A versatile group part weighting procedure is intended to underscore the significance of various gathering individuals, and stay away from the impact of malicious troupe individuals. Furthermore, the tests confirm elite of DCECP. Trials will be developed dependent using 15 genuine datasets as well as the system achieve accompanying ends. Appropriate parameter esteems will improve the presentation of the proposed methodology. The versatile troupe part weighting procedure assumes a significant job in DCECP.

In this paper, we suggest the two fold weighting semisupervised organization bunching depending upon chosen vital projection (DCECP) in order to cope with impediments of old semi-directed grouping outfit strategies.

(1) DCECP receives the irregular subspace strategy in blend with the requirement venture machine to perform highdimensional information bunching.

(2) System produces numerous subsets containing pairwise limitations that lower impact of such excess imperatives.

\section{REFERENCES}

1. Strehl, J. Ghosh, "Group Ensembles - A Knowledge Reuse Framework for Combining Multiple Partitions", Journal of Machine Learning Research, vol. 3, pp. 583-617, 2002.

2. T. Christou, "Coordination of Cluster Ensembles by means of Exact Methods", IEEE Transactions on Pattern Analysis and Machine Intelligence, vol. 33, no. 2, pp. 279-293, 2011.

3. N. Iam-On, T. Boongoen, S. Garrett, C. Value, "A Link-Based Approach to the Cluster Ensemble Problem", IEEE Transactions on Pattern Analysis and Machine Intelligence, vol. 33, no. 12, pp. 2396 2409, 2011.

4. Z. Yu, L. Li, H.- S. Wong, J. You, G. Han, Y. Gao, G. Yu, "Probabilistic Cluster Structure Ensemble", Information Sciences, vol. 267, pp. 16-34, 2014.

5. Rosni Ramle, "A Process of Wireless Sensor Network Using Maximum Energy Efficiency of Clustering Analysis", International Journal of Computing and Mathematics, 3(2), 2019. 